\title{
Valoración del Patrimonio Arquitectónico a través de la Tecnología Digital
}

\author{
Martínez Vergara Guillermo ${ }^{\mathrm{a}^{*}}$ \\ a Programa de Maestría y Doctorado en Arquitectura, Maestría en Arquitectura (opción Tecnología), Universidad Nacional Autónoma de México - UNAM, Ciudad \\ Universitaria, Ciudad de México - CDMX.
}

\begin{abstract}
Resumen
Cada vez son más notorios los efectos de la globalización en la sociedad, factores como la economía, la migración e incluso el sorprendente avance tecnológico de los últimos años, han ido cambiando poco a poco la percepción social de la población. Dichos cambios no pueden ser condenados debido a que marcan de manera significativa la evolución del ser humano y permiten el intercambio de ideas a través de todo el mundo. El problema surge cuando los habitantes de una región comienzan a ser indiferentes con sus propias ideologías y posturas culturales, trayendo como consecuencia una homogenización cultural que en muchas ocasiones concluye con el descuido y destrucción del patrimonio arquitectónico de las regiones. Ésta problemática es más notoria en las pequeñas localidades del país al enfrentarnos a un factor demográfico importante que es la migración; cada vez son más las personas que salen de su lugar de origen en busca de mejores oportunidades de calidad de vida, dejando a estos sitios vulnerables a la pérdida de patrimonio cultural. Si bien es cierto que el avance tecnológico ha atacado en contra de la integridad cultural, podemos aprovechar el interés actual de toda la población hacia este factor para dar un enfoque responsivo a la tecnología y utilizarla a favor del cuidado y protección de los elementos culturales y arquitectónicos con valor patrimonial por parte de los habitantes.
\end{abstract}

Palabras Clave: Patrimonio, tecnología, conservación, aplicación móvil, cultura

\section{Introducción}

El estudio del patrimonio arquitectónico trae una serie de beneficios que garantizan un mayor conocimiento de nuestra historia, aportando datos descriptivos sobre la forma de pensar y actuar de nuestras antiguas sociedades; permite además difundir los aspectos culturales que nos definen como personas pertenecientes a una cultura colectiva.

Los efectos de la globalización y la era tecnológica, incluyen un cambio en la forma de pensar de todas las naciones debido a que estos factores facilitan el intercambio social, económico, tecnológico y cultural, ocasionando de esta manera, que las sociedades generen conductas distintas y desarrollen una mentalidad multicultural. (Chonchol, 1998)

La sociedad mexicana no queda exenta de este fenómeno, la constante relación con naciones foráneas, trae consigo una fuerte pérdida de identidad cultural; la indiferencia por parte del mexicano hacia su cultura, está provocando el descuido del patrimonio arquitectónico, conduciéndolo hacia la destrucción, modificación y saqueo descontrolado.

La situación empeora en las pequeñas localidades del país debido a la migración excesiva por parte de los habitantes de estas poblaciones, cada vez es mayor el número de personas que sale de su lugar de origen en busca de oportunidades de empleo, educación y salud, dejando propensas a las regiones del país al descuido y destrucción de su arquitectura.

Factores como la globalización y el desarrollo tecnológico, no pueden ser detenidos ni erradicados porque se han convertido en un estilo de vida al que la sociedad se ha ido acostumbrando y adoptando como elementos que forman parte vital del desarrollo de nuestras actividades diarias.

La preocupación por la conservación del patrimonio arquitectónico y la sensibilidad del ser humano con su identidad cultural obliga a cambiar de enfoque a los elementos que están atentando contra la integridad cultural. La difusión de la conservación del patrimonio arquitectónico dirigido a los ciudadanos con el objetivo de lograr que se identifiquen y sensibilicen con los elementos que componen su presente y su pasado, se ha trabajado desde hace varios años a través de una serie de métodos que se han presentado especialmente para esta función apostando principalmente por las nuevas tecnologías como medio para lograr este fin.

\section{Posturas culturales}

A lo largo de los últimos años, cambios económicos, tecnológicos, sociales e incluso políticos, han transformado las culturas. Las sociedades pertenecientes a diferentes naciones del mundo. Podemos determinar, que la cultura abarca símbolos, significados, valores, instituciones, conductas y todos sus derivados, estos elementos a su vez, caracterizan sólidamente a una población humana, identificándola y distinguiéndola de las demás sociedades. La palabra cultura por sí misma, lleva consigo su propio peso de asociaciones en lenguajes y tradiciones diferentes.

En su libro, Culture and development, el autor Shanker, define principalmente tres componentes que construyen a una cultura:

\footnotetext{
* Autor en correspondencia.

Correos electrónicos: memo.vergara@hotmail.com (Martínez Vergara Guillermo),
} 
- Un sistema de valores significativos y normativos que establezcan un sentido de existencia y pertenencia del ser humano a una cultura colectiva, y que a su vez estipule las reglas sociales a seguir en dicha cultura.

- Una serie de elementos compartidos que incluya factores que hagan que la gente se identifique como miembro de un grupo como el territorio, el leguaje y la historia.

- La determinación por parte del individuo para ser identificado como parte fundamental de esa comunidad. (Shanker, 1998).

Bajo esta percepción, los componentes propuestos por Shanker se pueden resumir en cuatro principales elementos que debe tener una cultura para considerarse como tal: valores, normas, historia e identidad cultural. La clasificación de dichos elementos es simple y fácil de comprender, podemos hablar de un sistema de valores y reglas establecidos en un territorio con historia y lenguaje; sin embargo, la ausencia de una identidad cultural por parte del individuo, que le permita sentirse miembro activo e importante de una comunidad, anula o debilita la solidez de la estructura cultural de la sociedad.

Desde el comienzo de la observación del deterioro y destrucción de nuestros elementos culturales y arquitectónicos, se visualizaron los efectos de la globalización como factores principales que propiciaban esta problemática cultural. Se trata de una revolución no solamente social, sino que todos estos cambios introdujeron una nueva mentalidad en los individuos, lo cual ocasionó un cambio radical en sus intereses. Los efectos que causan la globalización mundial establecen diferentes puntos de vista a través de los cuales se puede identificar el principal impacto que tiene la cultura de las regiones en su desarrollo nacional e internacional. (Shanker, 1998). Con esta postura podemos deducir que, hablar de globalización no debe ser cuestión de condena para la cultura, la globalización es un fenómeno que no estamos en espera de que pase, sino que actualmente lo estamos viviendo y sus repercusiones en la vida diaria son evidentes. Dicho fenómeno no puede ser detenido, por lo cual, la adaptación a sus efectos se hace indispensable para la sobrevivencia cultural.

Esta connotación implica observar que la noción de patrimonio arquitectónico como concepto, no es estática sino por el contrario, está basada en un dinamismo que la misma cultura le marca. (Núñez 2013) por lo tanto, los efectos de la globalización y avance tecnológico, deben dejar de verse como factores amenazantes hacia nuestra cultura, en cambio debe tomarse un sentido responsivo hacia estos fenómenos, que en lugar de perjudicar a nuestros elementos culturales, valoremos su importancia como herramientas potenciales para lograr su conservación.

La tecnología es ahora parte del ambiente humano, y como tal, está estrechamente ligada y forma parte de los componentes que conforman nuestra cultura, los medios de comunicación en masa, la computadora y dispositivos móviles unen al mundo a través de sus redes, pero eliminan lo que es específico y retan la sobrevivencia de las culturas que son el corazón de todas las sociedades.

\subsection{Tecnología dentro del patrimonio arquitectónico}

El uso de las nuevas tecnologías relacionadas con el patrimonio cultural y arquitectónico, es un tema que cuenta con un largo historial de propuestas y estudios que han posicionado y analizado la factibilidad del uso de las herramientas tecnológicas aplicadas en los elementos que conforman nuestro patrimonio cultural.

El estudio de las herramientas tecnológicas como sustento para el patrimonio cultural y arquitectónico, comienza a partir de la incursión del sorprendente avance tecnológico que se ha observado durante los últimos años y que han favorecido cabalmente las necesidades que demanda el mundo globalizado en los diferentes sectores que estructuran a la sociedad actual.

La aplicación de la tecnología hacia los diferentes elementos que componen nuestra cultura, se han desarrollado y aplicado con la finalidad de optimizar y prevenir las distintas problemáticas por las que atraviesa actualmente el patrimonio cultural y arquitectónico. Podemos establecer entonces, que dentro de la temática de nuestro patrimonio cultural existen varios aspectos que tomar en cuenta para lograr su conservación.

La participación de la tecnología dentro de este aspecto siempre ha sido con diferentes metas, pero que a final de cuentas, tienen el objetivo de solucionar las problemáticas actuales que se han desarrollado en torno al patrimonio como lo son: la restauración, difusión, educación, exploraciones, reconstrucciones entre muchos otros aspectos comprendiendo éstos temas de difusión como la acción que permite poner en contacto a los individuos con su patrimonio, a adquirir mecanismos de interpretación que les ayude a comprender a estos hechos u objetos. (Rico, 2010)

La irrupción de las nuevas tecnologías como instrumento de conocimiento del Patrimonio Cultural es muy reciente, en poco más de dos décadas de aplicación se confirma su gran utilidad como soporte de la difusión y la investigación patrimonial." (Gómez, Quirosa, 2009). La visualización y análisis de todos estos recursos tecnológicos actuales nos llevó directamente a la propuesta del uso del internet como herramienta disponible en la mayoría de los lugares, siendo el causante de la eficacia de comunicaciones y relaciones entre distintos aspectos de nuestro país. El uso del internet se ha desarrollado y mejorado en los últimos años, su uso se ha vuelto tan indispensable, que hoy en día es inimaginable pensar como sería la vida sin este recurso.

Detrás de la invención, desarrollo y mejoramiento del internet viene el sorprendente avance de los dispositivos móviles, que debido a la portabilidad y conexión directa a la red, se puede decir que estas dos herramientas tecnológicas han revolucionado completamente la forma en la que los seres humanos vivimos, ésta situación ha creado una nueva mentalidad y forma de vida, en la que el dispositivo móvil pasa a ser una extensión artificial de nuestra persona. Los dispositivos móviles son utilizados a diario por millones de usuarios de todo el mundo, convirtiéndose en piezas fundamentales de la vida digital moderna (Luca, 2013). Hoy en día podemos asegurar que la mayoría de las personas no conciben el mundo real sin la utilización diaria de estos dos elementos, lo cual nos indica el interés y gusto por parte de los individuos hacia estas herramientas y nos permite abordar el tema desde esta perspectiva.

La utilización de los dispositivos móviles, especialmente los nuevos modelos de teléfonos inteligentes combinados con el despliegue del mercado de las aplicaciones móviles permiten al usuario a acceder a redes infinitas de información de una forma inmediata (Martínez y López, 2014) 
La propuesta de investigación, plantea y propone la creación de una nueva herramienta tecnológica utilizando a los dispositivos móviles como conducto para que a través de su funcionamiento y utilización, promueva la interacción de los individuos con su patrimonio cultural y propicie de esta manera el cuidado de los elementos culturales con el fin de evitar su destrucción y modificación descontrolada.

El producto final es una herramienta materializada en una aplicación móvil que resulte fácil de utilizar y que requiera recursos mínimos que se encuentren al alcance de la mayoría de las personas, de esta forma se pretende que la herramienta tecnológica llegue a una gran cantidad de la población sin caer en las problemáticas que se presentan en algunas de las propuestas tecnológicas anteriores como lo son: la falta de equipo, problemas de software o que implique a los individuos que se trasladen a un lugar en específico para poder hacer uso de ellas.

\subsection{Descripción del área de estudio}

Se lleva a cabo aplicación de la herramienta tecnológica en una localidad que sirva como caso de estudio para la evaluación de su efectividad. La localidad seleccionada debe contar con las características necesarias que indiquen y demuestren una problemática en la comunidad que haga necesaria la implementación de la aplicación móvil para analizar en qué medida se soluciona el problema. De acuerdo con las características solicitadas se selecciona como caso de estudio la comunidad de Cuitzeo Michoacán.

El desarrollo del municipio de Cuitzeo implica mejorar las condiciones de vida de su población con relación a; salud, educación, empleo y los servicios básicos para vivir una vida digna, es por todo lo anterior que el actual $\mathrm{H}$. Ayuntamiento de Cuitzeo Michoacán esta implementado este Plan de Desarrollo Municipal; donde se hace primeramente un verdadero diagnostico participativo en todo el municipio para conocer la realidad de los problemas y poder marcar las líneas estratégicas dentro del Plan de Desarrollo. Dentro del análisis diagnóstico de problemáticas en el municipio de Cuitzeo se identifican cuatro problemáticas que sustentan la elección del municipio de Cuitzeo como caso estudio para la aplicación

del prototipo tecnológico:

1. Falta de impulso del turismo en localidades con potencial.

2. Emigración excesiva.

3. Falta de un centro de cultura y deporte.

4. Falta de mejoramiento de la imagen urbana de área de los monumentos históricos, zona arqueológica y centros turísticos.

Sumado a esto, el Plan de Desarrollo Municipal de Cuitzeo posiciona a la emigración excesiva como un problema actual que se está desarrollando en el municipio y que con el transcurso de los años, en lugar de ir disminuyendo, se ha ido aumentando las cifras de las personas que salen del municipio en busca de mejores oportunidades de empleo, educación y vivienda. Esto está ocasionando entre muchas cosas, que la población del municipio se aleje de su lugar de origen y se descuiden los elementos culturales que distinguen a las diferentes regiones que componen a la comunidad de Cuitzeo.

El PDMC también menciona que en la actualidad, el municipio no cuenta con un centro de cultura, por lo cual la cultura del municipio se encuentra desprotegida al no existir una organización o programa que fomente la protección y cuidado de los elementos culturales.

A pesar de que el producto de la investigación no es meramente un centro de cultura, la temática está relacionada debido a que ambas estrategias tienen como finalidad, la difusión de la cultura del municipio para lograr la protección de los elementos arquitectónicos y culturales, que a través del tiempo han sido descuidados por parte de la población del municipio.

\subsection{Método de desarrollo de aplicación móvil}

La definición de un método que guíe el proceso de la creación de la aplicación móvil es indispensable, permite el correcto camino de la aplicación desde la conceptualización hasta la ejecución de la misma, debido a esto se establecen tres etapas que sigue el proceso de diseño para llegar al producto final.

\section{Pre- Producción.}

\section{Conceptualización}

El objetivo de esta etapa es tener una idea clara de la aplicación que pretendemos desarrollar, debe ser un proyecto que responda a las necesidades exigidas por los usuarios. La primera idea responde a una serie de documentación e investigación preliminar. (Cuello, Vittone, 2013)

$$
\begin{aligned}
& \text { - Ideación } \\
& \text { - Investigación } \\
& \text { - Formalización de la idea. }
\end{aligned}
$$

Definición

En este paso se describe a detalle el perfil de los usuarios hacia quienes va dirigida la aplicación móvil, además es en este punto en donde se sientan las bases para su funcionalidad lo que da como resultado un acercamiento al alcance que tiene el proyecto y a la complejidad de diseño.

- Definición de usuarios

- Definición funcional

2. Producción

Diseño

Dentro de ésta etapa se materializan los conceptos de la pre-producción, donde se crea un primer prototipo en forma de wireframe para ser probada por los usuarios y posteriormente realizar el diseño visual que permita la programación del código. Se presenta también la creación del isotipo, conceptualizado de ésta manera debido a que es la representación gráfica de un símbolo que ejemplifica la aplicación sin mencionarla completamente. (García 2015)
- Creación de isotipo
- Wireframe
- Prototipo
- Test con usuarios
- Diagrama de navegación 
- Guion técnico por pantalla

- Interfaz gráfica

- Diseño visual

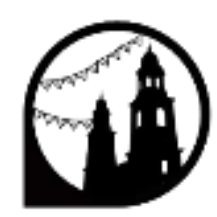

Ilustración 1. Isotipo de la aplicación móvil

Desarrollo

En esta etapa se animan los diseños y se crea la estructura funcional de la aplicación móvil. Una vez realizada la primera versión, se corrigen errores funcionales que garanticen la correcta ejecución para su aprobación en las diferentes tiendas.

- Programación de código

- Corrección de bugs

3.Post-producción

\section{Publicación}

Se realiza el lanzamiento de la aplicación móvil y se pone a disposición de los usuarios en tiendas. A partir del lanzamiento se lleva a cabo un seguimiento que a través de estadísticas y comentarios se visualiza el funcionamiento de la aplicación para corregir errores y realizar mejoras y actualizaciones.

- Lanzamiento

- Seguimiento

- Actualización

Los dispositivos móviles son utilizados a diario por millones de usuarios de todo el mundo, convirtiéndose en piezas fundamentales de la vida digital moderna (Luca, 2012) dicha aseveración justifica el medio utilizado para el objetivo de la investigación y plantea una forma diferente de ver al mundo digital a través de pequeños artefactos que abren las puertas a nuevas oportunidades y tareas que pueden ser realizadas a partir de un objeto común.

La funcionalidad del concepto de la aplicación móvil se resume en el siguiente esquema:

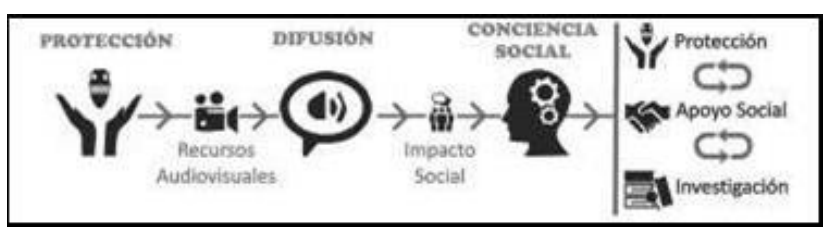

Ilustración 2. Esquema de conceptualización básica de funcionamiento de la herramienta tecnológica

A partir de la problemática y la preocupación por preservar el patrimonio arquitectónico y cultural de las pequeñas localidades del país, la investigación brinda una herramienta digital lo suficientemente atractiva para la sociedad actual que cuente con los recursos audiovisuales necesarios para que a través de esta se logre una difusión de la importancia del cuidado del patrimonio. La difusión busca crear un impacto dentro de las sociedades pertenecientes al patrimonio cultural arquitectónico que concluya en una concientización por parte de los habitantes y genere la cultura de la protección del patrimonio a través de apoyo social y proyectos de investigación.

\subsection{Flujo de información en la herramienta tecnológica}

Dentro de la planificación del flujo no entramos de lleno al diseño, es una primera visualización de las pantallas necesarias y su conexión entre sí. Es la manera gráfica de describir la disponibilidad del flujo y la forma en la que se relacionan cada uno de los elementos que se proponen.

Es en este momento cuando las ideas se transforman al papel sin entrar en detalles minuciosos de diseño, únicamente la definición de la estructura de las bases de usabilidad, funcionalidad y navegabilidad.

Cuando los usuarios ingresan por primera vez a un sitio o a una aplicación móvil, por lo general tratan de comprenderla sin recurrir a ayuda adicional, utilizando los conocimientos que han adquirido con anterioridad en plataformas similares, lo cual nos compromete a no realizar cambios profundos en la estructura de los sitios que lleguen a provocar que el usuario se sienta perdido dentro de la interacción del sitio y conduzca al fracaso de la aplicación. Debido a esto, es importante brindar al usuario herramientas con elementos y recursos con los que ya esté familiarizado, basando en eso el diseño de las pantallas e íconos que forman parte de la aplicación móvil.

Los íconos mostrados en el primer acercamiento con la aplicación son los correspondientes a la pantalla de registro, por sí solos los íconos son intuitivos por su forma y se deduce la información que solicita cada espacio en blanco: nombre, correo, teléfono, contraseña y la confirmación de contraseña, a pesar de esto fue necesario especificar con palabras lo que cada rubro solicita, ya que sin registrarte no puedes hacer uso de la aplicación móvil. Los íconos utilizados no son muy diferentes a los empleados en otras plataformas para solicitar datos, lo cual cumple con el objetivo de ser familiares para los usuarios y tener un primer encuentro amigable con la interfaz. 1.La primera pantalla que se muestra al momento de descargar la aplicación móvil se trata de una solicitud de datos básicos para poder registrarte y hacer uso de la misma. Se procura que en el registro se soliciten los datos más básicos con la finalidad de no alterar la tranquilidad del usuario y generar desconfianza al pedir datos más específicos. Al momento en el que pides de más datos, los usuarios comienzan a tener actitudes de desconfianza y prefieren interrumpir el proceso del registro. En el proceso de registro únicamente se piden los siguientes datos:

- Nombre de usuario

- Correo electrónico

- Teléfono

- Contraseña de seis dígitos.

Posterior a la solicitud de datos, la aplicación te lleva a una cláusula en donde los usuarios tienen que aceptar los Términos y Condiciones de Uso así como los acuerdos de Licencia en 
donde se estipulan las generalidades de la aplicación móvil así como las restricciones que tiene al momento de su uso.

2.Una vez aceptados los Términos y Condiciones de Uso, una tercera pantalla te lleva a iniciar sesión en la aplicación utilizando únicamente tu correo electrónico y la contraseña establecida.

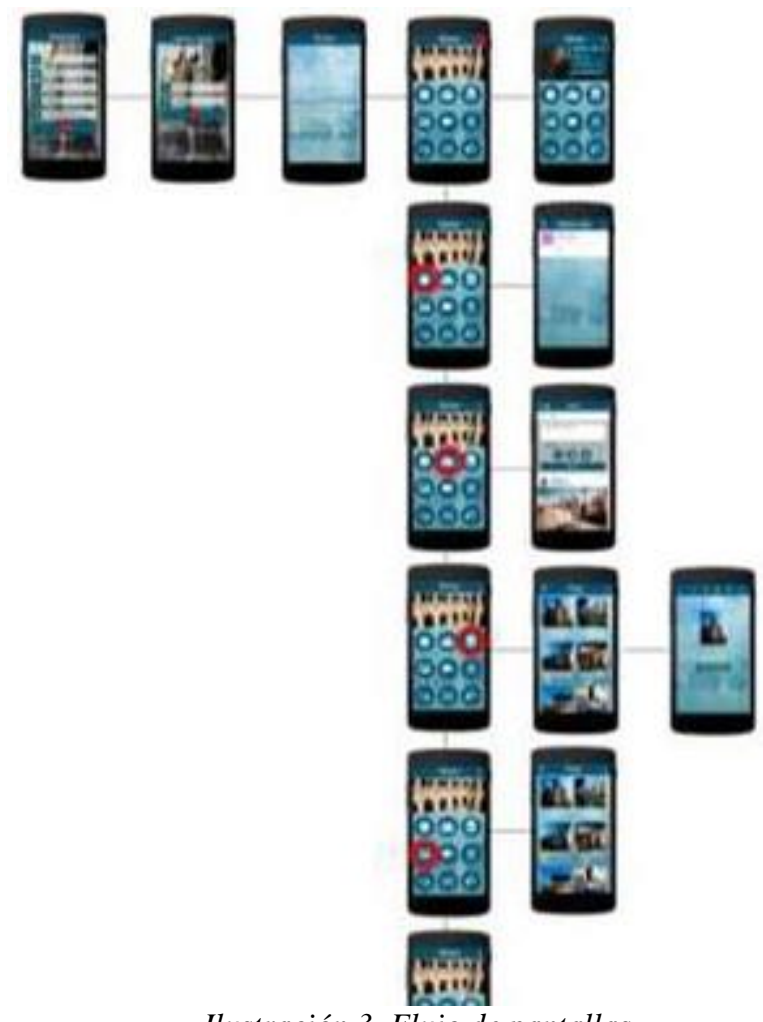

Ilustración 3. Flujo de pantallas

Cuando se inicia sesión, la aplicación te lleva a la pantalla de inicio o pantalla principal. En la pantalla de inicio se cuentan con todas las funciones que están dentro de la aplicación. Dentro de esta estructura se cuida un diseño limpio libre de elementos que puedan confundir la funcionalidad de la herramienta, se opta por un diseño intuitivo, en donde el usuario es capaz de reconocer las funciones de cada botón debido a que se trata de íconos utilizados en otras aplicaciones con los que el usuario ya está familiarizado.

Las funciones básicas con las que cuenta la aplicación móvil son:

- Barra de notificaciones

- Vinculación con página de Facebook

- Red social

- Información arquitectónica

- Foto-galería

- Video

- Calendario de eventos

- Comentarios

- Mapa con sitios de interés

- Encuesta

Pantalla de registro
1

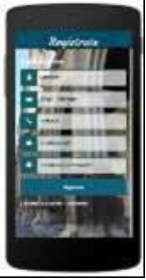

Ilustración 4. Pantalla de inicio de aplicación móvil

Cada una de estas funciones te lleva a una pantalla diferente en donde el usuario puede comenzar a interactuar con la información de la aplicación. La estructura de la aplicación está planificada dependiendo del grado de interactividad que el usuario puede tener con la función. En una primera etapa, en donde se encuentran la vinculación a Facebook, la red social y la sección de información arquitectónica es un espacio que el propio usuario de la aplicación móvil construye, en dichos espacios las personas pueden elegir el contenido de su interés que desean publicar o consultar.

En una segunda etapa, hablando de la fotogalería, la sección de videos y calendarios de eventos, es un espacio en donde el administrador de la aplicación decide la información que será publicada desde el punto de vista estratégico para captar la atención de los usuarios de la aplicación móvil.

Dentro de la tercera etapa, la sección de comentarios, mapa con sitios de interés, y la encuesta, es el espacio de consulta para los usuarios donde pueden expresar sus opiniones o consultar los lugares con valor patrimonial de su comunidad.

\section{Resultados y discusión}

En la presente investigación, se realiza el análisis de la relación que se tiene entre la identidad cultural de los habitantes de la comunidad de Cuitzeo y la valoración de su patrimonio arquitectónico. Es evidente que las variables manejadas en la investigación son de carácter cualitativo, lo cual resulta un poco complicado de evaluar debido a que nos enfrentamos a la parte subjetiva de la investigación, es decir, las variables están completamente ligadas a la percepción del grupo de individuos que van a ser evaluados para comprobar los resultados del proyecto.

Esto nos obliga a buscar la manera en la que los resultados cualitativos obtenidos a partir de la evaluación de la identidad cultural de los habitantes se puedan expresar cuantitativamente para lograr comprobar la validación del producto de investigación. Al tratar de evaluar la valoración del patrimonio arquitectónico y la relación que se tiene con la identidad cultural de sus habitantes, nos enfrentamos a conceptos bastante complejos debido a que no existe un número o un valor estandarizado que nos indique el nivel de sentido de identidad cultural que es óptimo en una sociedad.

Todas estas condiciones de la complejidad de los conceptos que estamos manejando, nos conduce a concluir que el ejercicio de evaluar la identidad cultural de las personas no es una tarea fácil, se necesita un instrumento anteriormente validado que nos proporcione resultados confiables sobre lo que se está evaluando.

Debido a esto, se propone la aplicación de una encuesta realizada con el objetivo de conocer a profundidad el sentimiento de valoración de los habitantes posterior al uso de la aplicación móvil. Se trata de una serie de preguntas concisas 
que tratan los puntos de interactividad, interés y apropiación de la aplicación móvil por parte de los usuarios. Gracias a la facilidad y accesibilidad de la aplicación, la encuesta fue realizada dentro de la misma, en donde se detallan y reflejan de forma automatizada las respuestas de los usuarios y teniendo un análisis estadístico preliminar de los resultados. Se optó por la realización de la encuesta por éste medio debido a que los usuarios ya estaban familiarizados con el uso de la aplicación móvil y resulta práctico utilizar la misma herramienta tecnológica para evaluarse a sí misma.

\section{Conclusión}

A pesar de tener dependencias y leyes encargadas de salvaguardar el patrimonio arquitectónico, la destrucción y modificación de sitios y monumentos sigue ocurriendo de manera descontrolada por parte de los habitantes del lugar, conforme ha avanzado la tecnología en el ámbito social, se han ido desarrollando estrategias tecnológicas que fusionan la efectividad de la tecnología con el patrimonio cultural, logrando el interés por parte de las personas.

Los métodos utilizados en la actualidad para evaluar la usabilidad de un software no establecen una guía o modelo evaluativo con la suficiente precisión para determinar el éxito o fracaso de la usabilidad de cómo medirla incluyendo las aplicaciones móviles, el uso de las aplicaciones móviles siempre estará estrechamente influenciada y ligada por el contexto en donde se utilice, lo que quiere decir que intervienen diversos factores que alteran los resultados.

La implementación de una aplicación móvil especializada en un contexto y lugar en específico resulta ser interesante y atraer la atención de los habitantes logrando su interés en la conservación y cuidado de su patrimonio arquitectónico, al brindar una herramienta que engloba los principales intereses de los individuos, en este caso una aplicación móvil dentro de un contexto social y festivo, se desarrolla un sentido de apropiación por parte de los habitantes hacia la herramienta tecnológica.

Además de los factores tecnológicos, existen eventualidades que de manera involuntaria consolidan la identidad cultural de la sociedad, los incidentes causados por el sismo el día 19 de septiembre de 2017 en el país demostraron poder unir a los seres humanos como una sociedad sensible a su cultura, fortaleciendo una empatía hacia su lugar de origen y los elementos arquitectónicos que lo conforman.

A pesar de que un desastre natural demostró que el uso de la tecnología no es el único medio existente para lograr la consolidación de la identidad cultural de las personas, se posiciona como una herramienta poderosa, actual y fácil de utilizar que actúa en gran medida como intermediaria para que los individuos interactúen con su patrimonio arquitectónico, dándole de esta manera el carácter responsivo del que se habla al principio de la investigación.

El hecho de no ser el único medio nos aventura a seguir explorando las posibilidades y estrategias que se pueden llevar a cabo para seguir realizando la difícil tarea de conservación y valoración del patrimonio arquitectónico.

\section{English Summary}

\author{
Valuation of architectural heritage through digital \\ technology.
}

\section{Abstract}

The effects of globalization on society are becoming more noticeable, factors such as the economy, migration and even the surprising technological advance of recent years have been changing the social perception of the population little by little. These changes cannot be condemned because they significantly mark the evolution of the human being and allow the exchange of ideas throughout the world.

The problem arises when the inhabitants of a region begin to be indifferent with their own ideologies and cultural positions, bringing consequently a cultural homogenization that in many cases ends with the neglect and destruction of the architectural heritage of the regions. This problem is more evident in the small towns of the country when facing an important demographic factor that is migration; more and more people leave their place of origin in search of better opportunities for quality of life, leaving these sites vulnerable to the loss of cultural heritage.

While it is true that technological progress has attacked cultural integrity, we can take advantage of the current interest of the entire population towards this factor to give a responsive approach to technology and use it in favor of the care and protection of cultural elements and architectural with heritage value by the inhabitants.

\section{Keywords:}

Heritage, technology, conservation, app, culture

\section{Agradecimientos}

El presente trabajo es una investigación interdisciplinaria en la cual se empleó la asesoría de expertos en los diferentes temas tratados siendo dirigido por la Dra. Genevieve Lucet perteneciente al Instituto de Investigaciones Estéticas de la UNAM, así como la significativa participación como asesores teóricos y tecnológicos de la Mtra. Karla Erazo Castrejón perteneciente a la Dirección General de Cómputo y de Tecnologías de Información y Comunicación y el Dr. Ivan San Martín Córdova, investigador de tiempo completo de la Facultad de Arquitectura de la UNAM

\section{Referencias}

Chonchol J. (1998). Impacto de la globalización en las sociedades latinoamericanas: ¿qué hacer frente a ello?, scielo [en línea] https://goo.gl/1XLkTZ

Cuello, J. y Vittonoe, J. (2013) Diseñando apps para móviles 18-20

De Luca, D. (2013) Apps HTML5 para móviles: Desarrollo de aplicaciones para smartphones y tablets basados en tecnologías web, $15-30$

García, J. Técnicas de diseño gráfico corporativo (2014) 33-35

Gómez, L. y Quirosa V. (2009). Nuevas Tecnologías para Difundir el Patrimonio Cultural: Las reconstrucciones virtuales en España e-rph Junio 2009.

Martínez, T. y López, V. (2014), La tecnología móvil: de medio de comunicación a instrumento educativo y de inclusión 39-46 
Núñez, Angélica (2013). Resignificaciones y reapropiaciones del patrimonio cultural. En Baukara 4, Bitácoras de antropología e historia de la antropología en América Latina, 137 pp, (p.6-21)

Rico, L. (2003) La difusión del patrimonio en la educación no formal. Un estudio de caso sobre los materiales curriculares de los Gabinetes Pedagógicos de Bellas Artes, Trabajo para la suficiencia
Investigadora, Facultad de Filosofía y Letras, Departamento de Historia del Arte, Universidad de Málaga

Shanker, R. (1998) Culture and development. International Development Information Centre. Development Express. Canada Communication Group. Westby, C. \& Atencio, D.J. (2002). Computers, culture, and learning. Top Land Disord, 22. (4), 70-87. 